\title{
Threat Evaluation of Early Warning Detection Based on Incomplete Attribute Information TODIM Method
}

\author{
Zhiyang Gao, Li Zhu, Zheng Li, Pengcheng Fan \\ Electronic Engineer Institute.Hefei,China.230037 \\ gaozhiyangtougao@163.com
}

Keyword: Incomplete attribute information; TODIM method; Early warning detection; Threat assessment.

\begin{abstract}
The incomplete attribute information TODIM method is applied to the early warning detection threat evaluation to enhance decision-making scientific and targeted due to the limitations of traditional multi-attribute evaluation methods. This paper introduces the incomplete attribute information TODIM method on the basis of critical thinking and analysis steps combining with the characteristics of early warning detection system, establishes the evaluation index system of early warning detection system threats, expounds the process of threat assessment and verifies the validity and rationality of this method according to case simulation.
\end{abstract}

\section{Introduction}

Threat assessment of early warning detection which is a complicated Multiple Attribute Decision Making (MADM) problem, with series of uncertain factors need to be solved scientifically and properly. The method of TODIM originated from Tomada de decisao interativa e multicritévio in Portuguese means interactive multiple attribute decision making. By means of calculating the superiority of a certain target than others', this method reorders and selects targets according to the description of experts' experience .Its purpose is to provide answers for decision making. Regular TODIM method requires that attribute information should be expressed completely .However, experts could hardly provide complete attribute information among all kinds of decision making problems. They are more likely to provide incomplete attribute information which causes these kinds of problems hardly to be solved. Incomplete attribute information TODIM method whose conclusions are more objective and practical by defining incomplete attribute matrices, calculating superiority and reordering target superiority extends and develops the method of MADM. Therefore, the method is applied into threat assessment of early warning detection.

\section{Brief Introduction of Incomplete Attribute Information TODIM Method}

Incomplete attribute information TODIM method is used to solve MADM problems which include two types: interval number attribution and attribution concluded by method Delphi. Incomplete attribute information TODIM method need eight steps: 1.Defining incomplete attribute information. This method need to define the scope of incomplete attribute information which means scope represents the concept of incomletence.2.Standardizing decision making matrices. MADM problems are usually divided into two types of benefit and cost which is often standardized into benefit type for convenient. This paper only researches threat assessment of early warning detection which should be classified into benefit types. Therefore, the step of standardization could be omitted in it.3. Calculating related attribute weight. Related attribute weight means the ratios between each target attribute weight and the maximum weight of all the target attribute.4.Calculating the superiority matrices of each target attribute relative to others.Superiority is extended as relative threat degree.5.Calculating superiority matrices in total. In this paper, superiority matrices are extended as threat degree matrices.6. Determining target functions and constraint condition models.7.Solving the optimal model.8.Reordering targets according to threat degree in total. 


\section{Threat Assessment of Early Warning Detection Index System}

The operational capability of threat assessment of early warning detection system is mainly determined by functions, operation missions, deployment and battlefield survivability. This paper defines that all the early warning detection systems are the same kind detecting our side, which means that the threat of early warning detection systems are just determined by operation capability of detection system. According to operation, detecting area, detecting precision, timeliness, numbers of detecting targets and analyzing targets, detecting period and econtermeasure (ECM ) capability are selected as threat assessment indices.

Detecting Area. Detecting area is a significant index of early warning detection system, which means the largest area of detecting certain target at certain detecting probability. The detecting areas of different detection systems are various due to their platforms, missions and operational capacities. Generally speaking, the larger detecting area the more detailed information of our operation movements and targets they will get to know, the more threaten we will possess.

Detecting Precision. Detecting precision means the error range between the true values from targets and measured values from detection systems, which is the maximum measured values of statistical averages. Generally, different detecting precision of the detection system is various, the detection precision is higher, the higher resolution precision of target.

Time Sensitiveness. Time sensitiveness is a kind of index of early warning detection systems whose time from detection to acquision. The time of early warning detection systems needs to be reduced as much as possible from detection to get all the process of target information and the total process of transport. Different early warning detection systems, aiming at different application levels, also have its specific timeliness. A general sense that the stronger the timeliness, the higher the threat level.

Numbers of Detecting Targets and Analyzing Targets. The numbers of detecting targets and analyzing targets means early warning detection systems detect and analyze the number of targets at the same time. The larger the number is, the greater probability of detection will be and the same batch of targets will be more likely destroyed.

Detecting Period. Detecting period means the time from early warning detection systems first time detecting targets to second time identifying same targets. Generally speaking, the second time identifying targets means the targets lose their capability of concealment. The shorter detecting period is, the higher threat will be during the operation.

Econtermeasure (ECM) Capability. Econtermeasure (ECM) capability is an important operational integrated index when operation forces are used in the EW environment, which is both a technical problem and a tactical problem. The capability is related with the electronic means of attack, electronic attack strategy, the performance of early warning detection system and operational characteristics. The higher ECM capability, the stronger anti electronic jamming and anti entity destroy resistance ability will be and the more threat to our sides will be.

\section{Procedure of Early Warning Detection Threat Assessment}

Provide Early Warning Detection System Indexes. Early warning detection system indexes mainly comes from materials and technology investigation intelligence, whose index system has set up in the last section. In this article, the method of Delphi is adopted to quantize parameters. The basic procedure of Delphi method is: setting goal, selecting exporters, designing evaluation sheets and feeding back experts opinions. Specifically, after the opinions of experts are collected, statistic data are collected, integrated, counted and then fed back to experts anonymously. This procedure will recycle several times until the results are convergent and consistent. Flow chart of Delphi method is showed as Picture 1. 


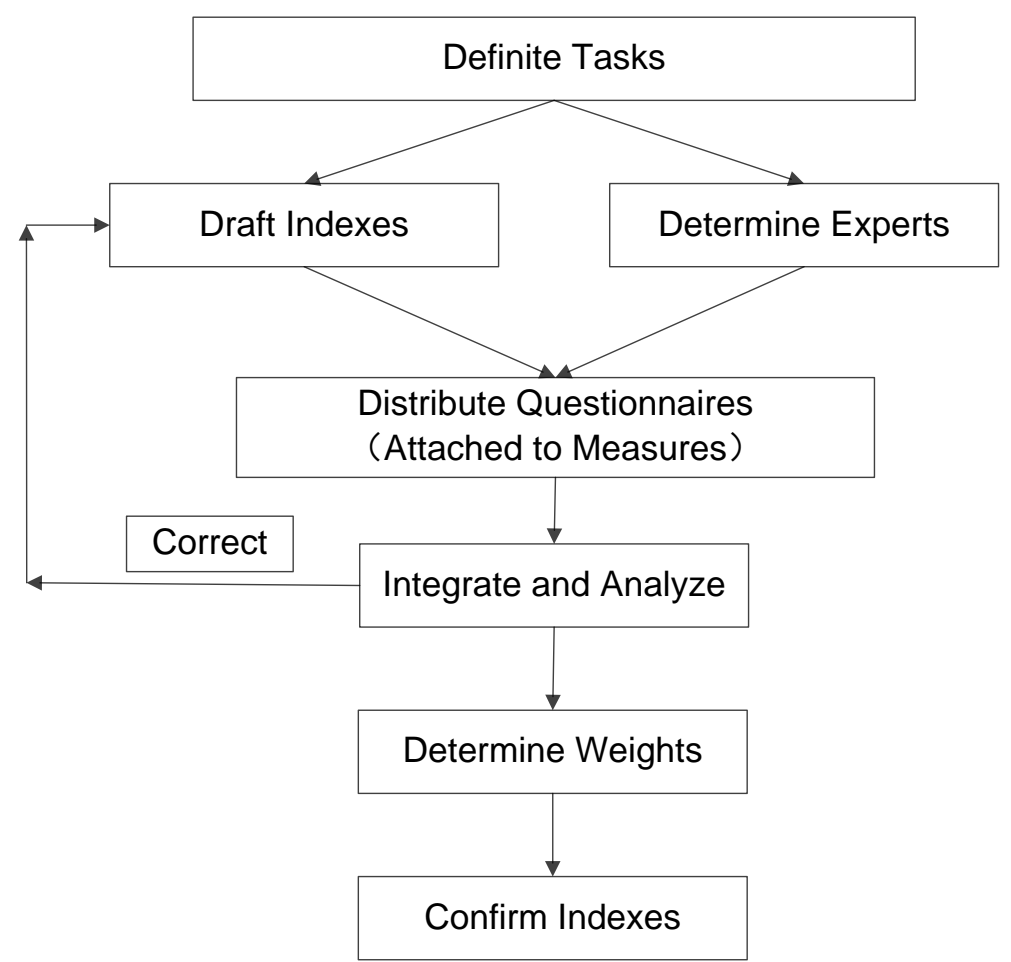

Picture 1 Flow Chart of Delphi Method

Indexes of early warning detection systems are provided by means of standard tables which are showed as Table 1.

Table1 Indexes

\begin{tabular}{|c|c|c|c|c|c|c|c|}
\hline Platform & $\begin{array}{l}\text { Serial } \\
\text { Number }\end{array}$ & $\begin{array}{l}\text { Detecting } \\
\text { Area }\end{array}$ & $\begin{array}{l}\text { Detecting } \\
\text { Precision }\end{array}$ & $\begin{array}{c}\text { Time } \\
\text { Sensitiveness }\end{array}$ & $\begin{array}{c}\text { Numbers } \\
\text { of } \\
\text { Detecting } \\
\text { Targets } \\
\text { and } \\
\text { Analyzing } \\
\text { Targets } \\
\end{array}$ & $\begin{array}{l}\text { Detecting } \\
\text { Period }\end{array}$ & $\begin{array}{c}\text { ECM } \\
\text { Capability }\end{array}$ \\
\hline Base X & $M_{1}$ & $\beta_{11}$ & $\beta_{12}$ & $\beta_{13}$ & $\beta_{14}$ & $\beta_{15}$ & $\beta_{16}$ \\
\hline Base X & $M_{2}$ & $\beta_{21}$ & $\beta_{22}$ & $\beta_{23}$ & $\beta_{24}$ & $\beta_{25}$ & $\beta_{26}$ \\
\hline Base X & $\ldots .$. & & & $\cdots$ & & & \\
\hline Base X & $M_{x}$ & $\beta_{x 1}$ & $\beta_{x 2}$ & $\beta_{x 3}$ & $\beta_{x 4}$ & $\beta_{x 5}$ & $\beta_{x 6}$ \\
\hline
\end{tabular}

Quantize Indexes of Early Warning Detection Systems. According to system indexes, experts adopt the method of Delphi to quantize indexes by their own experience and system abilities. Compared to the method of TODIM, attribute information ought to be replaced as threaten degree. Specially, $M_{1}, M_{2}, \cdots, M_{x}$ represent code numbers of early warning detecting systems; $N_{1}, N_{2}, N_{3}, N_{4}, N_{5}, N_{6}$ represent detecting area, detecting precision, time sensitiveness, numbers of detecting targets and analyzing targets, detecting period and ECM capability respectively(platform is qualitative index which need not be quantized). $a_{11}$ represents detecting area threaten degree of $M_{1}$ early warning detecting system. $a_{12}$ represents detecting precision threaten degree of $M_{1}$ early warning detecting system. The rest can be done in the same 
manner .Then $a_{x 6}$ represents ECM capability threaten degree of $M_{1}$ early warning detecting system. Data range of $a_{x y}$ is $[0,1]$ which means experts should evaluate index in this range.

Table 2 Attribute Information Description

\begin{tabular}{ccccc}
\hline & \multicolumn{3}{c}{ Attribute Information } \\
\cline { 2 - 5 } Targets & $N_{1}$ & $N_{2}$ & $\ldots \ldots$. & $N_{y}$ \\
\hline$M_{1}$ & $a_{11}$ & $a_{12}$ & $\ldots \ldots$. & $a_{1 y}$ \\
$M_{2}$ & $a_{21}$ & $a_{21}$ & $a_{2 y}$ \\
$\ldots \ldots$ & & & $\ldots \ldots$ & \\
$M_{x}$ & $a_{x 1}$ & $a_{x 2}$ & $\ldots \ldots$. & $a_{x y}$ \\
\hline
\end{tabular}

Since the circumscribed knowledge and ability of experts, experts could not quantify all the indexes precisely. A part of threaten degrees are showed as Formula (1), Formula (2) and Formula (3).

$$
\begin{gathered}
a_{x y} \geq \lambda a_{z y}, x, z \in I, x \neq z, y \in J, \lambda \in R^{+} \\
\alpha^{-} \leq a_{x y} \leq \alpha^{+}, x \in I, y \in J, \alpha^{-}, \alpha^{+} \in R^{+} \\
u^{-} a_{z y} \leq a_{x y} \leq u^{+} a_{z y}, x, z \in I, x \neq z, y \in J, u^{-}, u^{+} \in R^{+}
\end{gathered}
$$

Confirm Relative Threaten Degree Weight of Early Warning System. According to different early warning detection system, attribute weight vectors represent six different attribute weights which are showed as $w=\left(w_{1}, w_{2}, \cdots w_{6}\right)^{T}$. Data range is [1,7]. Reference vectors $w^{*}$ could be calculated out by $w^{*}=\max \left\{w_{1}, w_{2}, \cdots, w_{j}\right\}$.And then, relevant weight vectors $w_{y}{ }^{\prime}$ is calculated out through $w_{y}^{\prime}=\frac{w_{y}}{w^{*}}$.

Calculate Relevant Threaten Degree and Totality Threaten Degree of Each Early Warning Detection System. In common incomplete attribute information TODIM method, superiority is used to represented virtues or defect degree. This article evaluates early warning detection system with the help of the method. Therefore, superiority is comprehended as relevant threaten degree. Higher relevant threaten degree will cause higher danger to our side. According to Formula (3), $s_{y}\left(M_{x}, M_{z}\right)$ represents the relevant threaten degree of $M_{x}$ system compared to $M_{z}$ system on $N_{y}$ attribute information.

$$
s_{y}\left(M_{x}, M_{z}\right)=\left\{\begin{array}{cc}
\sqrt{\frac{a_{x y}-a_{z y}}{a_{y}^{+}-a_{y}^{-}} w_{y}^{\prime} / \sum_{y=1}^{j} w_{y}^{\prime},} & a_{x y}-a_{z y}>0 \\
0 & a_{x y}-a_{z y}=0 \\
-\frac{1}{\theta} \sqrt{\frac{a_{z y}-a_{x y}}{a_{y}^{+}-a_{y}^{-}} \sum_{y=1}^{j} w_{y}^{\prime} / w_{y}^{\prime}}, & a_{x y}-a_{z y}<0
\end{array}\right.
$$

Introduction: $a_{y}{ }^{+}=\max \left\{a_{x y}\right\} a_{y}{ }^{+}=\min \left\{a_{x y}\right\} ; \theta$ represents attenuation coefficient which means experts' ability of avoiding making error decision. The value of $\frac{1}{\theta}$ is positive correlation with the ability of avoiding making errors. The range of $\theta$ is $0<\theta<\sum_{y=1}^{i} w_{y}^{\prime} / w_{y}^{\prime}$. 
According to Formula (4), $s\left(M_{x}, M_{z}\right)$ could be calculated out, which means the relevant threaten degree of $M_{x}$ system compared to the other systems on $N_{y}$ attribute information.

$$
s\left(M_{x}, M_{z}\right)=\sum_{y=1}^{i} s_{y}\left(M_{x}, M_{z}\right)
$$

Lastly, totality threaten degree of $M_{x}$ system could be calculated by Formula (5):

$$
g\left(M_{x}\right)=\sum_{z=1}^{i} s\left(M_{x}, M_{z}\right)
$$

Structure the Maximum Threaten Degree Selection Model of Early Warning Detection System. Totality threaten degree of early warning detection system is higher, which means more threaten factors to our side. Therefore, the maximum threaten degree selection model should be structured. According to Formula (6a), Formula (6b) and Formula (6c), constraints could be established. By means of MATLAB 2013a, the results can be calculated out.

$$
\begin{gathered}
T=\max \left\{g\left(M_{x}\right)\right\}, x \in I \\
\text { s.t. } a_{x y} \geq \lambda a_{z y}, x, z \in I, x \neq z, y \in J, \lambda \in R^{+} \\
\alpha^{-} \leq a_{x y} \leq \alpha^{+}, x \in I, y \in J, \alpha^{-}, \alpha^{+} \in R^{+} \\
u^{-} a_{z y} \leq a_{x y} \leq u^{+} a_{z y}, x, z \in I, x \neq z, y \in J, u^{-}, u^{+} \in R^{+}
\end{gathered}
$$

Rank Relevant Threaten Degree. According to Formula (7), totality threaten degree could be calculated out. The value of $F\left(M_{x}\right)$ is ranked from high to low value.

$$
F\left(M_{x}\right)=\frac{g\left(M_{x}\right)-\min _{x \in I}\left\{g\left(M_{x}\right)\right\}}{\max _{x \in I}\left\{g\left(M_{x}\right)\right\}-\min _{x \in I}\left\{g\left(M_{x}\right)\right\}}, \quad x \in I
$$

$\max _{x \in I}\left\{g\left(M_{x}\right)\right\}$ represents the totality maximum threaten degree of all the targets. On the

\begin{tabular}{|c|c|c|c|c|c|c|c|}
\hline Platform & $\begin{array}{l}\text { Serial } \\
\text { Number }\end{array}$ & Detecting Area & $\begin{array}{l}\text { Detecting } \\
\text { Precision }\end{array}$ & $\begin{array}{c}\text { Time } \\
\text { Sensitiveness }\end{array}$ & $\begin{array}{l}\text { Numbers } \\
\text { of } \\
\text { Detecting } \\
\text { Targets } \\
\text { and } \\
\text { Analyzing } \\
\text { Targets } \\
\end{array}$ & $\begin{array}{l}\text { Detecting } \\
\text { Period }\end{array}$ & $\begin{array}{c}\text { ECM } \\
\text { Capability }\end{array}$ \\
\hline Space-Based & $M_{1}$ & $225000 \mathrm{~km}^{2}$ & $0.7 \mathrm{~m} \sim 0.95 \mathrm{~m}$ & 30min & 320 & $18 \mathrm{~h}$ & weaker \\
\hline Space-Based & $M_{2}$ & $60000 \mathrm{~km}^{2}$ & $0.3 \mathrm{~m} \sim 1 \mathrm{~m}$ & $67 \mathrm{~min}$ & 450 & 89.5min & general \\
\hline Air-Based & $M_{3}$ & $40000 \mathrm{~km}^{2}$ & $0.2 \mathrm{~m} \sim 0.33 \mathrm{~m}$ & $15 \mathrm{~min}$ & 220 & $10 \mathrm{~s}$ & stronger \\
\hline Air-Based & $M_{4}$ & $50000 \mathrm{~km}^{2}$ & $0.3 \mathrm{~m} \sim 0.46 \mathrm{~m}$ & $8 \mathrm{~min}$ & 180 & $17 \mathrm{~s}$ & general \\
\hline Sea-Based & $M_{5}$ & $16816 \mathrm{~km}^{2}$ & $0.55 \mathrm{~m} \sim 0.73 \mathrm{~m}$ & $7 \mathrm{~min}$ & 195 & $14 \mathrm{~s}$ & weaker \\
\hline Ground-based & $M_{6}$ & $200000 \mathrm{~km}^{2}$ & $0.85 \mathrm{~m} \sim 1.2 \mathrm{~m}$ & $25 \mathrm{~min}$ & 440 & $57 s$ & weak \\
\hline
\end{tabular}
contrary, $\min _{x \in I}\left\{g\left(M_{x}\right)\right\}$ represents the totality minimum threaten degree of them. The values of $F\left(M_{x}\right)$ are corresponding with targets. Higher value means more dangerous to equipments.

\section{Case Simulation}

In certain environment, there are several early warning detection systems showed in Table 3.Task is to select the early warning detection system with the maximum threaten to us.

Table 3 Parameters of Early Warning Detection Systems 
The paper uses the method of Delphi to evaluate the task above to improve the reliability of decision-making and get the result more precisely. However, since the task is jammed for time. Parts of attribute information are represented as incomplete style, which are showed in Table 4 below.

Table 4 Experts’ Evaluation Result of Early Warning Detection Systems

\begin{tabular}{|c|c|c|c|c|c|c|c|}
\hline \multirow[t]{2}{*}{$\begin{array}{l}\text { Platfor } \\
\text { m }\end{array}$} & \multirow{2}{*}{$\begin{array}{c}\text { Seria } \\
1 \\
\text { Num } \\
\text { ber }\end{array}$} & Detecting Area & $\begin{array}{l}\text { Detecting } \\
\text { Precision }\end{array}$ & $\begin{array}{c}\text { Time } \\
\text { Sensitiveness }\end{array}$ & $\begin{array}{l}\text { Numbers of } \\
\text { Detecting } \\
\text { Targets and } \\
\text { Analyzing } \\
\text { Targets } \\
\end{array}$ & $\begin{array}{l}\text { Detecting } \\
\text { Period }\end{array}$ & \multirow[t]{2}{*}{$\begin{array}{c}\text { ECM } \\
\text { Capability }\end{array}$} \\
\hline & & $N_{1}$ & $N_{2}$ & $N_{3}$ & $N_{4}$ & $N_{5}$ & \\
\hline $\begin{array}{c}\text { Space-B } \\
\text { ased }\end{array}$ & $M_{1}$ & $a_{11}=0.83$ & $a_{62}<a_{12}<1$ & $0.65<a_{13}<$ & $a_{14}=0.73$ & $a_{15}=0.24$ & $a_{16}=0.34$ \\
\hline $\begin{array}{c}\text { Space-B } \\
\text { ased }\end{array}$ & $M_{2}$ & $a_{21}=0.53$ & $a_{22}=0.57$ & $a_{23}=0.95$ & $a_{64}<a_{24}<$ & $a_{25}=0.36$ & $a_{16}<a_{26}<0$ \\
\hline $\begin{array}{c}\text { Air-Bas } \\
\text { ed }\end{array}$ & $M_{3}$ & $a_{51}<a_{31}<a_{41}$ & $a_{32}=0.86$ & $a_{33}=0.58$ & $a_{34}=0.8 a_{64}$ & $a_{35}=0.89$ & $0.83<a_{36}<$ \\
\hline $\begin{array}{l}\text { Air-Bas } \\
\text { ed }\end{array}$ & $M_{4}$ & $0.4<a_{41}<0.9$ & $a_{12}<a_{42}<0$ & $a_{43}=0.41$ & $a_{44}=0.66$ & $a_{45}=0.83$ & $a_{56}<a_{46}<\mathrm{a}$ \\
\hline $\begin{array}{c}\text { Sea-Bas } \\
\text { ed }\end{array}$ & $M_{5}$ & $0.2<a_{51}<0.3$ & $a_{52}=0.44$ & $0.39<a_{53}<$ & $0.66<a_{54}<$ & $a_{55}=0.93$ & $a_{56}=0.36$ \\
\hline $\begin{array}{c}\text { Ground- } \\
\text { based }\end{array}$ & $M_{6}$ & $a_{31}<a_{61}<0.8$ & $0.30<a_{62}<$ & $\mathrm{a}_{13}<a_{63}<1$ & $a_{54}<a_{64}<$ & $a_{65}=0.68$ & $a_{66}=0.4$ \\
\hline
\end{tabular}

According to what experts have provided, weight vectors might be calculated as $w=(4,6,2,7,3,1)^{T} \quad$.Therefore, reference weight can be represent as $w^{*}=\max \left\{w_{1}, w_{2}, w_{3}, w_{4}, w_{5}, w_{6}\right\}=w_{4}=7$. Then relevant weight vector is $w^{\prime}=\left(\frac{4}{7}, \frac{6}{7}, \frac{2}{7}, 1, \frac{3}{7}, \frac{1}{7}\right)^{T}$.

After what has mentioned above, according to Formula 5, Formula 5 (a), Formula 5(b) and Formula 5(c), optimization model and constraints could be acquired.

$$
\begin{gathered}
T=\max \left\{\sum_{z=1}^{6} \sum_{y=1}^{6} s_{y}\left(M_{x}, M_{z}\right)\right\} \\
\text { s.t. }\left\{\begin{array}{cccccc}
a_{11}=0.83 & a_{62}<a_{12}<a_{52} & 0.65<a_{13}<0.67 & a_{14}=0.73 & a_{15}=0.24 & a_{16}=0.34 \\
a_{21}=0.53 & a_{22}=0.57 & a_{23}=0.95 & a_{64}<a_{24}<0.93 & a_{25}=0.36 & a_{26}=0.55 \\
a_{51}<a_{31}<a_{41} & a_{32}=0.86 & a_{33}=0.58 & a_{34}=1.1 a_{54} & a_{35}=0.89 & a_{26}<a_{36}<0.87 \\
0.4<a_{41}<0.97 a_{21} & a_{62}<a_{42}<0.88 a_{32} & a_{43}=0.41 & a_{44}=0.66 & a_{45}=0.82 & a_{56}<a_{46}<\mathrm{a}_{26} \\
0.2<a_{51}<0.32 & a_{52}=0.44 & 0.39<a_{53}<a_{13} & a_{44}<a_{54}<a_{34} & a_{55}=0.93 & a_{56}=0.36 \\
a_{11}<a_{61}<0.88 & 0.30<a_{62}<0.34 & a_{13}<a_{63}<0.75 & a_{64}=0.87 & a_{65}=0.68 & a_{66}=0.4
\end{array}\right.
\end{gathered}
$$


Decision matrix $A=\left[a_{x y}\right]_{6 \times 6}$ could be acquired through calculating the optimization model by means of MATLAB 2013a.

$$
A=\left[\begin{array}{llllll}
0.830 & 0.300 & 0.650 & 0.730 & 0.240 & 0.340 \\
0.530 & 0.570 & 0.950 & 0.930 & 0.360 & 0.840 \\
0.400 & 0.830 & 0.580 & 0.696 & 0.890 & 0.874 \\
0.514 & 0.730 & 0.410 & 0.660 & 0.830 & 0.769 \\
0.200 & 0.440 & 0.403 & 0.636 & 0.930 & 0.360 \\
0.880 & 0.340 & 0.750 & 0.870 & 0.680 & 0.400
\end{array}\right]
$$

According to Formula 3, relevant threaten degree matrix of each two systems could be acquired.

$$
s=\left[\begin{array}{cccccc}
0 & -12.4494 & -10.1038 & -9.0495 & -4.4331 & -8.7586 \\
-0.4196 & 0 & -4.7740 & -2.8576 & -1.2721 & -3.1444 \\
-3.1761 & -5.4716 & 0 & -0.3374 & 0.5283 & -5.1355 \\
-4.4343 & -7.6293 & -7.0092 & 0 & -0.1632 & -5.9983 \\
-5.7130 & -13.7522 & -11.7688 & -9.1539 & 0 & -8.6842 \\
1.1185 & -9.1282 & -8.0704 & -6.9012 & -1.6592 & 0
\end{array}\right]
$$

According to Formula 4, totality relevant threaten degree could be acquired, which means that $g\left(M_{1}\right)$ represents the totality threaten degree of $M_{1}$ system compared to the others. $g\left(M_{2}\right), g\left(M_{3}\right), g\left(M_{4}\right), g\left(M_{5}\right)$ and $g\left(M_{6}\right)$ could be expressed as same meaning.

$$
\begin{aligned}
& g=\left[g\left(M_{1}\right), g\left(M_{2}\right), g\left(M_{3}\right), g\left(M_{4}\right), g\left(M_{5}\right), g\left(M_{6}\right)\right] \\
& =[-44.7944,-12.4677,-13.5924,-25.2343,-49.0721,-24.6405]
\end{aligned}
$$

According to Formula 6, relevant threaten degree could be standardized into ranking matrix for convenient to compare with each other.

$$
F=\left[F\left(M_{1}\right), F\left(M_{2}\right), F\left(M_{3}\right), F\left(M_{4}\right), F\left(M_{5}\right), F\left(M_{6}\right),\right]=[0.1169,1,0.9693,0.6512,0,0.6674] \quad \text { Six }
$$
systems which cause different levels of threaten to our side are showed in the picture below.

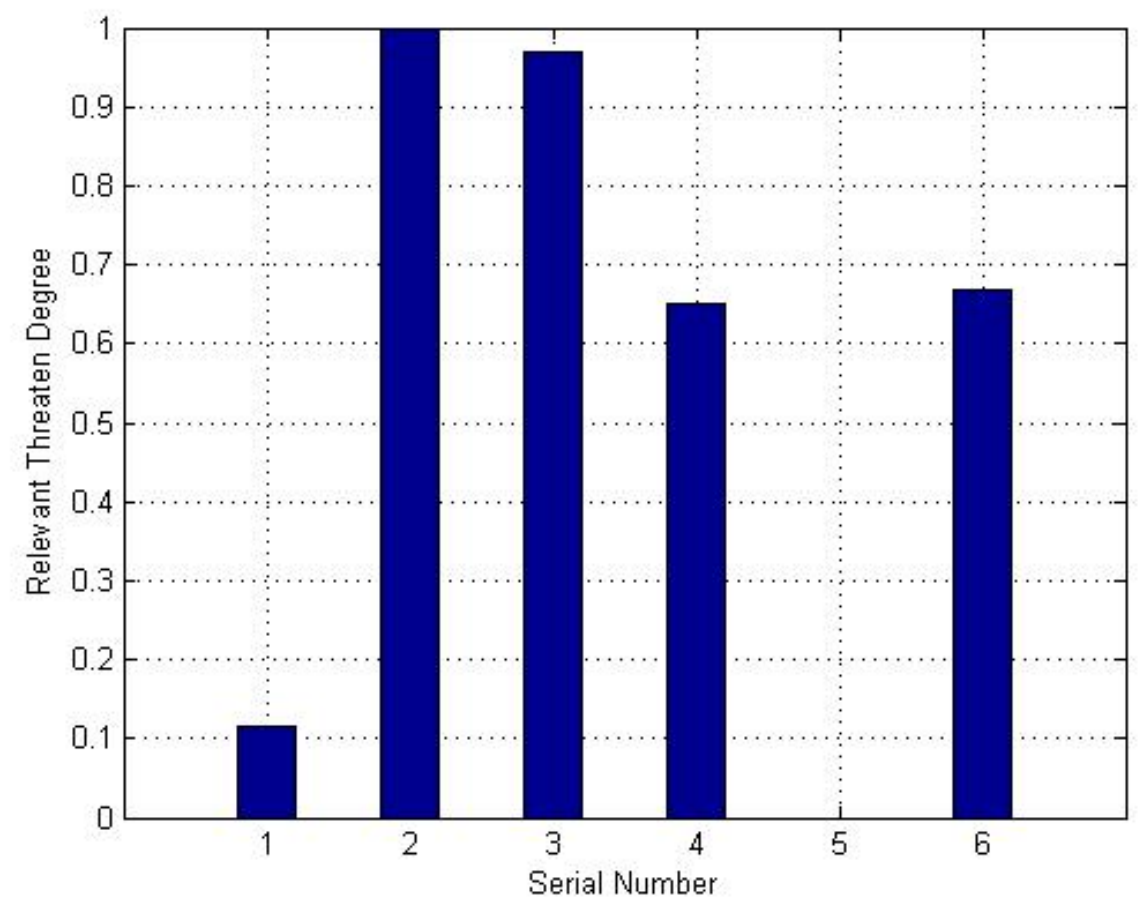

Picture 2 Threaten Degree Rank of Early Warning Detection System 
Conclusion: $M_{2}>M_{3}>M_{6}>M_{4}>M_{1}>M_{5}$ represents the rank of six early warning detection system.

\section{Reference}

[1] J.Q. Wang, .E. Li. TODIM method with multi-value neutrosophic sets.Control and Decision, 2015(1):2.

[2] Y.P. Jiang, X. Liang. OPERATIONS RESEARCH AND MANAGEMENT SCIENCE, 2015(2):117.

[3] Hwang C.L., Yoon K. Multiple attribute decision making: Methods and Applications.New York:Springer-Verlag,1981.

[4] Peide Liu, Fei Teng, An Extended TODIM Method for Multiple Attribute Group Decision-Making Based on 2-Dimension Uncertain Linguistic Variable .COMPLEXITY, 2014Wiley Periodicals:4-6.

[5] H.B. Jin, Z.h. Hua, S.B. Wan. Research on Evaluation System of the Joint Air Intelligence Early Warning and Surveillance System.Journal of Detection \&Control, 2009(12):46

[6] J.h. Wang, X.P. Wu. Effectiveness analysis of ship communication security equipment based on fuzzy synthetic evaluation. SHIP SCIENCE AND TECHNOLOGY, 2009, 31(4):103.

[7] Kahneman D, Kahneman D. Judgement under uncertainty: Heuristics and biases. Science, 1974, 185(4157):1124-1130.

[8] Bell D E. Regret in decision making under uncertainty.Operations Research, Journal of Economic Theory,1987,41(2):268-271. 